\title{
Fractional Differential Equation as a Models of Newton Fluids for Stress and Strain Problems
}

\author{
$1^{\text {st }}$ Endang Rusyaman \\ Departement of Mathematics, FMIPA \\ Universitas Padjadjaran \\ Bandung, Indonesia \\ rusyaman@unpad.ac.id
}

\author{
$2^{\text {nd }} D$. Chaerani \\ Departement of Mathematics, FMIPA \\ Universitas Padjadjaran \\ Bandung, Indonesia \\ d.chaerani@unpad.ac.id
}

\author{
$3^{\text {rd }}$ Kankan Parmikanti \\ Departement of Mathematics, FMIPA \\ Universitas Padjadjaran \\ Bandung, Indonesia \\ parmikanti@unpad.ac.id
}

\begin{abstract}
An ordinary differential equation is a branch of mathematics that is always interesting to be learned and developed due to its numerous variations in both the theory and its application. In general, the most discussed differential equation is the ordinary differential equation that has the natural number as its order. However, currently, the order of a differential equation which has been developed into a fractional-order (i.e. rational numbers) is also very interesting to be studied. This paper presents a study of ordinary differential equations that has fractional order, in which the left side contains two derivative functions with fractional order while the right side contains polynomial function $\boldsymbol{n}$ degree. Specifically, this paper presents the general form of equations, methods of finding the solution in three cases, and its application. Solutions are proposed using the Laplace transformation and its inverse and are expressed in the form of Mittag-Lefler function. Its graph is also later described using Matlab. Results of this research are expressed by three functions of three different theoretical cases and a solution to an application problem. Additionally, the study has also shown that the convergence of a number sequence of fractional differential equation order is positively related to the convergence of solution function sequence. There are many applications of fractional differential equations in the field of viscoelasticity. Therefore, at the end of the paper, this application is presented particularly regarding the relationship between stress and strain for solids and for Newtonian fluids.
\end{abstract}

Keywords-Fractional Differential equations, Mittag-Lefler, stress, Strain

\section{INTRODUCTION}

Fractional Calculus is the development of calculus in which rational numbers are used instead of natural numbers. Particularly, it is regarding the derivative and integral of a function where natural number as the order of derivative and integral becomes the rational number $\alpha$ as the order. As a matter of fact, this fractional calculus has existed for more than 300 years ago, when Leibniz sent a letter to L'Hospital in 1695 asking: "What would happen if the order of derivative is $1 / 2$ ?" [1]. This question inspired many other mathematicians in the 18th century such as Louiville, Riemann, and Weyl to develop fractional-ordered derivative. Furthermore, the fractional calculus development was continued by Fourier, Abel, Leibniz, Grünwald, and Letnikov, who had hugely contributed in this knowledge for years.

Nowadays, many scientists and engineers are interested to deepen and apply this knowledge in various fields such as D. Matignon who discussed fractional differential function in Web and System Theory, and Podlubny et al. who discussed fractional-ordered partial differential function [2-4]. Meanwhile, Adam Loverro used the fractional derivative in Physics Engineering regarding Mechanics and Aerodynamics, Yang Quan Chen et al. applied fractional calculus in Control Sciences and P. W. Ostalczyk who made a review about Grünwald-Letnikov fractional-order backward-difference [5-7].

Several mathematicians applied fractional calculus in the energy field. J. Wallner guarantees the existence of interpolation that can minimize energy [8]. Besides that, M. Hoffer and $\mathrm{H}$. Pottman designed energy-minimizing rigid body motions in the presence of obstacles [9]. Also, H. Gunawan et al. developed a fractional order and correlated it with material elasticity [10]. They found that the order of fractional differential equation can be associated with elasticity. A Comparable finding was observed by Nicole Heymans and Igor Podlubny with a series of example in viscoelasticity [11]. Their results showed that it was possible to associate physical meaning with the initial condition which is stated in Riemann-Liouville fractional derivatives. The last reference study is related with the stress and strain [12]. In that paper, Alberto Carpinteri and Pietro Cornetti used the local fractional calculus operators as a new mathematical tool to get the static and kinematic equations. The solutions obtained from the model are fractal, i.e. reproduce the side effects due to stress and strain localization [12].

This paper presents the existence and solution of the fractional differential function of:

$a_{n} y^{\left(a_{n}\right)}+a_{n-1} y^{\left(\alpha_{n-1}\right)}+\cdots+a_{1} y^{\left(a_{1}\right)}+a_{0} y-u(t)=0$ where $y^{(\alpha)}$ states fractional derivative of $y$ with respect $x$ with order $\alpha, a_{n}$ is the real constants, and $u(x)$ is function of $x$. In addition, the graphs of solution functions are presented to help in the understanding of the relation between the convergence of derivative-orders number sequence and the convergence of solution function sequence. Lastly, this 
paper presents the application of fractional differential function in viscoelasticity, particularly, shows the relationship between stress and strain through a fractional model.

\section{METHODS}

To solve the fractional differential equations, Laplace transforms and Mittag-Leffler functions are used. The following is the description.

\section{Fractional Derivative}

As mentioned before, the fractional derivative is the generalization result of the ordinary derivative with order natural number expanded into rational number $\alpha$. The derivation formula of fractional derivative function was presented differently by several mathematicians. RiemannLouiville defined fractional derivative with order $\alpha$ around $x$ $=a$ as follows,

$$
\begin{aligned}
& { }_{a} D_{x}^{\alpha} f(x)=\frac{1}{\Gamma(n-\infty)}\left(\frac{d}{d x}\right)^{n} \int_{a}^{x} f(t)(x-t)^{-\{\alpha-n ! 1)} d t \\
& \text { with } n-1 \leq \alpha<n \text { or } n-1=\lfloor\alpha\rfloor .
\end{aligned}
$$

Different from Riemann-Liouville, Grunwald-Letnikov defined fractional derivative of $f(x)$ with order $\alpha$ in interval $[a$ ,b] with

$$
D_{x}^{\alpha} f(x)=\lim _{\pi \rightarrow 0} \frac{1}{h^{\pi}} \sum_{\{=0}^{n}(-1)^{i} \frac{\Gamma(\alpha+1)}{\Gamma(i+1) \Gamma(\alpha-i+1)} f(x-i h)
$$

with $n=\left\lfloor\frac{b-a}{n}\right\rfloor$.

From (2), if $f(x)=x^{p}$, then $\alpha$-th derivative of $f(x)$, or derivative of $f(x)$ with order $\alpha$ to $x$ as follows:[8]

$$
D_{x}^{\alpha} x^{n}=\frac{\Gamma(p+1)}{\Gamma(p-\alpha+1)} x^{p-\alpha}
$$

\section{Special Functions}

It is known that Laplace transformation has an important role in solving Ordinary Differential Function matters. In this paper, it can be seen how effective Laplace transformation solves the differential equation with fractional-order, where the solution will be declared in the special form of function, namely Mittag-Leffler which has two parameters. So, in this section, we will present the definitions of Laplace transformation and the definitions of the Mittag-Lefler function with examples and their properties.

The Laplace transformation of function $f(t)$ is defined as follows:

$$
\mathrm{L}\{f(t)\}=F(s)=\int_{0}^{\infty} e^{-s t} f(t) d t
$$

On the contrary, $f(t)$ is the inverse of Laplace transformation of $F(s)$, denotated with

$$
\mathrm{L}^{-1}\{F(s)\}=f(t)
$$

Regarding derivative function, the Laplace transformation has a property that if $f(t)$ is a function that is differentiable $n$ times, then it implies

$$
\mathrm{L}\left\{f^{(n)}(t)\right\}=s^{n} \mathrm{~L}\{f(t)\}-s^{n-1} f(0)-s^{n-2} f^{\prime}(0)-\ldots-f^{(n-1)}(0) .
$$

If $D_{t}^{\alpha} f(t)$ denotes derivative of $f(t)$ with respect $t$ with fractional order $\alpha$, then the Laplace transformation with null initial condition is

$$
\mathrm{L}\left\{D_{t}^{\alpha} f(t) ; s\right\}=s^{\alpha} \mathrm{L}\{f(t)\}=s^{\alpha} F(s)
$$

Another important special function in fractional calculus is the Mittag-Leffler function which was introduced in 1953. The function is presented in $[6,7]$.

The Mittag-Leffler function which is a function with two parameters $\alpha$ and $\beta$ is

$$
E_{\alpha, \beta}(z)=\sum_{k=0}^{\infty} \frac{z^{k}}{\Gamma(\alpha k+\beta)} ; \alpha>0, \beta>0
$$

This function is very flexible, since by changing both parameters with constants will results in another different function. For example,

$$
\begin{aligned}
& \text { 1. } E_{1,1}(z)=\sum_{k=0}^{\infty} \frac{z^{k}}{\Gamma(k+1)}=\sum_{k=0}^{\infty} \frac{z^{k}}{k !}=e^{z} . \\
& \text { 2. } E_{2,1}\left(-z^{2}\right)=\sum_{k=0}^{\infty} \frac{\left(-z^{2}\right)^{k}}{\Gamma(2 k+1)}=\sum_{k=0}^{\infty} \frac{(-1)^{k} z^{2 k}}{(2 k) !}=\cos z . \\
& \text { 3. } E_{2,1}(z)=\sum_{k=0}^{\infty} \frac{z^{k}}{\Gamma(2 k+1)}=\sum_{k=0}^{\infty} \frac{(\sqrt{z})^{2 k}}{(2 k) !}=\cosh \sqrt{z} .
\end{aligned}
$$

If $\beta=1$, the Mittag-Leffler function becomes one parameter function which is

$$
E_{\alpha}(z) \equiv E_{\alpha, 1}(z)=\sum_{k=0}^{\infty} \frac{z^{k}}{\Gamma(\alpha k+1)} .
$$

Another function from Mittag-Leffler type in [3] and [4] is

$$
\varepsilon_{k}(t, \lambda ; \alpha, \beta)=t^{\alpha k+\beta-1} E_{\alpha, \beta}^{(k)}\left(\lambda t^{\alpha}\right),
$$

where $E_{\alpha, \beta}^{(k)}(z)$ is derivative $k$ from Mittag-Leffler function as follows

$$
E_{\alpha, \beta}^{(k)}(z)=\sum_{i=0}^{\infty} \frac{(i+k) ! z^{i}}{i ! \Gamma(\alpha i+\alpha k+\beta)} ; k=0,1,2, \cdots .
$$

The followings are some examples of Laplace transformation from several Mittag-Leffler functions. [4,5].

$$
\begin{aligned}
& \text { 1. } \mathrm{L}\left\{t^{\alpha-1} E_{\alpha, \alpha}\left(-\lambda t^{\alpha}\right)\right\}=\frac{1}{s^{\alpha}+\lambda}, \\
& \text { 2. } \mathrm{L}\left\{t^{\beta-1} E_{\alpha, \beta}\left(-\lambda t^{\alpha}\right)\right\}=\frac{s^{\alpha-\beta}}{s^{\alpha}+\lambda}, \text { and } \\
& \text { 3. } \mathrm{L}\left\{\varepsilon_{k}(t, \pm \lambda ; \alpha, \beta)\right\}=\frac{k ! s^{\alpha-\beta}}{\left(s^{\alpha} \mp \lambda\right)^{k+1}}
\end{aligned}
$$

\section{RESULT AND DISCUSSION}

The Fractional Differential Equation is a differential equation in which its derivative order is the fractional number. There are three forms presented in this paper. 
Case-1: $\quad a y^{(\alpha)}+b y=c$ with $1 \leq \alpha \leq 2$ and $c$ is real constant.

The solution of this differential equation is obtained by using Laplace transformation in each segment, results in

$$
\mathrm{L}\left\{a y^{(\alpha)}+b y\right\}=\mathrm{L}\{c\} .
$$

By using Laplace transformation lineary property, it is obtained

$$
a \mathrm{~L}\left\{y^{(\alpha)}\right\}+b \mathrm{~L}\{y\}=\mathrm{L}\{c\},
$$

Therefore, the equation (1) becomes

$a s^{\alpha} F(s)+b F(s)=\frac{c}{s}$, so that $F(s)\left(a s^{\alpha}+b\right)=\frac{c}{s}$ Thus, $F(s)=\frac{\frac{c}{a}}{s\left(s^{a}+\frac{b}{a}\right)}$

The general solution of $y(t)$ by using $(6)$ is

$$
y(t)=\mathrm{L}^{-1}\{F(s)\}=\mathrm{L}^{-1}\left\{\frac{c}{a} \frac{0 ! s^{-1}}{\left(s^{\alpha}+\frac{b}{a}\right)}\right\}=\frac{c}{a} \varepsilon_{0}\left(t,-\frac{b}{a} ; \alpha, \alpha+1\right) .
$$

In form of Mittag-Leffler function, by using (5), it could be obtained another form of $y(t)$ as follows

$$
y(t)=\frac{c}{a} t^{\alpha} E_{\alpha, \alpha+1}\left(-\frac{b}{a} t^{\alpha}\right) .
$$

Finally, based on the definition of Mittag-Leffler function, the solution of the fractional differential equation case-1 is

$$
y(t)=\frac{c}{a} t^{\alpha} \sum_{k=0}^{\infty} \frac{\left(-\frac{b}{a} t^{\alpha}\right)^{k}}{\Gamma(\alpha k+\alpha+1)}=\frac{c}{a} t^{\alpha} \sum_{k=0}^{\infty} \frac{\left(-\frac{b}{a} t^{\alpha}\right)^{k}}{(\alpha k+\alpha) !} .
$$

For example, for $\alpha=1$ with $a=2, b=1$, and $c=1$, and initial condition $y(0)=0$, the fractional differential equation solution is:

$$
\begin{gathered}
y(t)=\frac{1}{2} t^{2} \sum_{k=0}^{\infty} \frac{\left(-\frac{1}{2} t^{2}\right)^{k}}{(2 k+2) !}=\sum_{k=0}^{\infty} \frac{(-1)^{k}\left(\frac{1}{2} t^{2}\right)^{k+1}}{(2 k+2) !}=\sum_{k=1}^{\infty} \frac{(-1)^{k+1}\left(\frac{1}{2} \sqrt{2} t\right)^{2 k}}{(2 k) !} \\
=1-\cos \frac{1}{2} \sqrt{2} t
\end{gathered}
$$

As illustration, three graphs of solution function with order $\alpha$ are presented below.

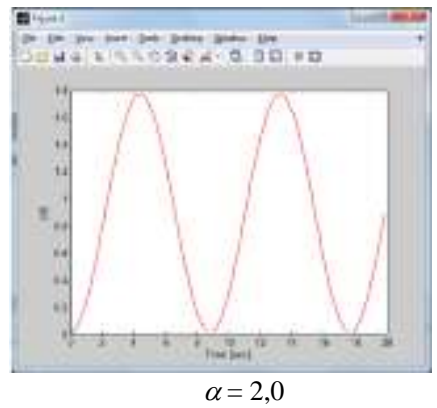

$\alpha=2,0$

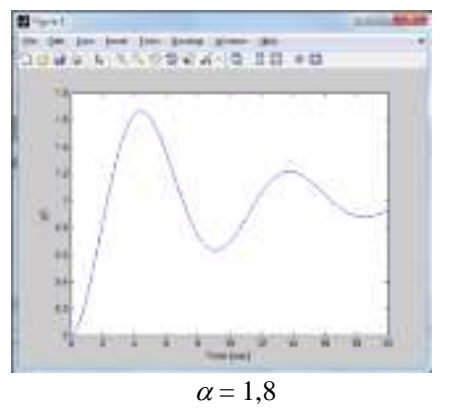

$\alpha=1,8$

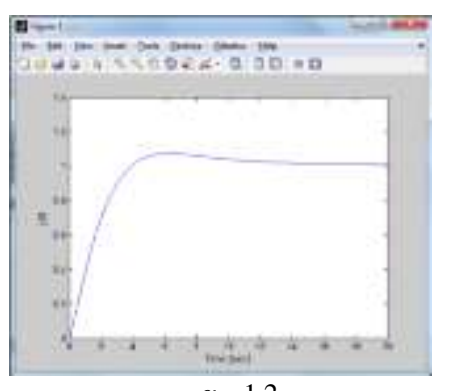

$\alpha=1,2$

Fig. 1. Graph of Solution Function Case-1

Case-2: The more general of fractional differential equation with order $(\alpha, \beta)$ is

$$
a y^{(\alpha)}+b y^{(\beta)}=u(t),
$$

where $\alpha>\beta, a$ and $b$ are real constants, and $u(t)$ is polynomial $\sum_{i=0}^{n} c_{i} t^{i}$. In the case of $u(t)$ is the function of exponent, logarithm, or trigonometry, thus, through Mac Laurin sequence, that function is converted into polinomial. By using Laplace transformation, it is obtained

$$
\mathrm{L}\left\{a y^{(\alpha)}+b y^{(\beta)}\right\}=\mathrm{L}\left\{\sum_{i=0}^{n} c_{i} t^{i}\right\}
$$

With Laplace transformation lineary property, it is resulted 


$$
a s^{\alpha} F(s)+b s^{\beta} F(s)=\sum_{i=0}^{n} \frac{c_{i}, i !}{s^{i+1}},
$$

Hence obtained

If we denote

$$
F_{i}(s)=\frac{\frac{c_{i}(i !)}{s^{i+1}}}{\left(a s^{\alpha}+b s^{\beta}\right)}, \quad i=0,1, \cdots, n,
$$

and $y_{i}(t)$ states the solution of $a y^{(\alpha)}+b y^{(\beta)}=c_{i} t^{i}$, then the general solution of the fractional differential equation (7) is

$y(t)=\sum_{t=0}^{n} y_{i}(t)=\sum_{t=0}^{n} \mathrm{~L}^{-1}\left(E_{t}(s)\right)=\sum_{t=0}^{n} \mathrm{~L}^{-1}\left(\frac{\frac{c_{1}(i)}{s^{i+1}}}{\left(\cos ^{a}+b s^{\natural)}\right.}\right) ; i=0,1, \cdots, n$.

The solution of $y_{i}(t)$ is $\mathrm{L}^{-1}\left\{F_{i}(s)\right\}$, thus could be stated as

$$
\begin{aligned}
& y_{i}(t)=\mathrm{L}^{-1}\left(\frac{\frac{c_{i}(i !)}{s^{i+1}}}{\left(a s^{\alpha}+b s^{\beta}\right)}\right)=\mathrm{L}^{-1}\left\{\frac{c_{i}(i !) s^{-i-1}}{a s^{\beta}\left(s^{\alpha-\beta}+\frac{b}{a}\right)}\right\} \\
& =\frac{c_{i}(i !)}{a} \mathrm{~L}^{-1}\left\{\frac{s^{-i-1-\beta}}{\left(s^{\alpha-\beta}+\frac{b}{a}\right)}\right\}=\frac{c_{i}(i !)}{a} \mathrm{~L}^{-1}\left\{\frac{0 ! s^{\alpha-\beta-(\alpha+i+1)}}{\left(s^{\alpha-\beta}+\frac{b}{a}\right)^{0+1}}\right\} .
\end{aligned}
$$

By using form (6) regarding Laplace transformation inverse, it is resulted

$$
y_{i}(t)=\frac{c_{i}(i !)}{a} \varepsilon_{0}\left(t,-\frac{b}{a} ; \alpha-\beta,(\alpha+i+1)\right) .
$$

Based on form (5), general solution could be stated as

$$
\begin{gathered}
y_{i}(t)=\frac{c_{i}(i !)}{a} t^{(\alpha-\beta) \cdot 0+\alpha+i+1-1} E_{\alpha-\beta_{r}(\alpha+i+1)}\left(-\frac{b}{a} t^{(\alpha-\beta)}\right) \\
=\frac{c_{i}(i !)}{a} t^{\alpha+i} E_{\alpha-\beta_{n}(\alpha+i+1)}\left(-\frac{b}{a} t^{(\alpha-\beta)}\right) .
\end{gathered}
$$

Furthermore by using the definition of Mittag-Leffler function, it is obtained

$$
\begin{gathered}
y_{i}(t)=\frac{c_{i}(i !)}{a} t^{\alpha+i} \sum_{k=0}^{\infty} \frac{\left(-\frac{b}{a} t^{(\alpha-\beta)}\right)^{k}}{\Gamma((\alpha-\beta) k+\alpha+i+1)} \\
=\frac{c_{i}(i !)}{a} t^{\alpha+i} \sum_{k=0}^{\infty} \frac{\left(-\frac{b}{a} t^{(\alpha-\beta)}\right)^{k}}{((\alpha-\beta) k+\alpha+i) !}
\end{gathered}
$$

For example, the solution of fractional differential equation

$$
y^{(\alpha)}+2 y^{(\beta)}=1+2 t+t^{2} .
$$

With order $(\alpha, \beta)=\left(\frac{5}{3}, \frac{1}{2}\right)$ and initial conditions $f(0)=0$ and $f^{\prime}(0)=0$, the solution is

$$
\begin{gathered}
y(t)=y_{0}(t)+y_{1}(t)+y_{2}(t) \\
=t^{\frac{5}{3}} \sum_{k=0}^{\infty} \frac{\left(-2 t^{\frac{7}{6}}\right)^{k}}{\left(\frac{7}{6} k+\frac{5}{3}\right) !}+2 t^{\frac{8}{3}} \sum_{k=0}^{\infty} \frac{\left(-2 t^{\frac{7}{6}}\right)^{k}}{\left(\frac{7}{6} k+\frac{8}{3}\right) !}+2 t^{\frac{11}{3}} \sum_{k=0}^{\infty} \frac{\left(-2 t^{\frac{7}{6}}\right)^{k}}{\left(\frac{7}{6} k+\frac{11}{3}\right) !}
\end{gathered}
$$


Graphs of the solution function with order $(\alpha, \beta)$ can be seen in Fig. 2 below.

\section{Model of Stress and Strain Problem}

Application of fractional differential equations may cover various fields, including dynamical system, control theory, signal processing and others. This paper shows how the order of fractional differential equations are used in determining the stress and strain in solid and fluid.

The fFluid flow consists of two classifications. The first one is a Newtonian fluid, a fluid which has a linear curve on the coordinates with stress as the vertical axis and the strain as the horizontal axis. Hence the ratio between stress and strain, also known as fluid viscosity, is constant. The uniqueness of Newtonian fluid is that the fluid will continue to flow even if there are forces acting on the fluid. This is because the viscosity of a Newtonian fluid does not change when there are forces acting on the fluid. Thus the viscosity of a Newtonian fluid depends only on the temperature and pressure, for example, water, blood, and honey. The different characteristic will be found in the non-Newtonian fluid, a fluid which experiences viscosity change when there are forces acting on the fluid. This means that the non-Newtonian fluid does not have a constant viscosity. The example of this type is mortar, mud, and soy sauce.

If $\delta(t)$ states stress and $\varepsilon(t)$ expresses strain which both are dependent on the time $t$, then Newtonian-fluid model is

$$
\delta(t)=\mu \frac{d \varepsilon(t)}{\mathrm{d} t}
$$

where $\mu$ is the coefficient of viscosity.

Aside from the fluid, in case of solid material, it is also known as the term of elasticity, in which if this material is given a stress then it will experience stretching/relaxation. In this case, it satisfies Hooke's law

$$
\delta(t)=E \varepsilon(t)
$$

where $E=\frac{\text { stress }}{\text { strain }}$ is elasticity or the Young's modulus.

The stress expression then can be transformed to time domain as a fractional differential equations as

$$
\delta(t)=E \tau^{\alpha} \frac{d^{\alpha} \varepsilon(t)}{d t^{\alpha}}
$$

Here $\tau$ is ratio of the shear viscosity to Young's modulus, $\alpha$ is the rational numbers between 0 and 1 , where $\alpha=0$ represents the Hooke's Law and $\alpha=1$ represents stress and strain for a Newtonian fluid. Indeed, if for $\alpha=0$ we have the elasticity and for $\alpha=1$ we have viscosity. Thus for the fractional, we have viscoelasticity. For $1 \leq \alpha \leq 2$, it illustrates the oscillation process which is strongly associated with the fractional order $\alpha$ and natural frequency.

Generally, the fractional differential equation model for relaxation problem that equivalent to the model (9) is:

$$
\varepsilon^{(\alpha)}(t)+A \varepsilon(t)=\delta(t)
$$

where $0 \leq \alpha \leq 1$, initial condition $\varepsilon^{(k)}(0)=\varepsilon_{0}^{(k)}, A$ is relaxation coefficient, $\delta(t)$ is stress and $\varepsilon(t)$ expressed strain.

\section{Example:}

Given a fractional Differential equation on relaxation problem $\varepsilon^{(0.5)}(t)+2 \varepsilon(t)=t \sin t$.

By using Maclaurin series, obtained another form as

$$
\varepsilon^{(0.5)}(t)+2 \varepsilon(t)=\sum_{i=1}^{\infty} \frac{(-1)^{i+1}}{(2 i-1) !} t^{2 i}
$$

From (8), solution of this fractional differential equation is

$$
\varepsilon(t)=\sum_{i=1}^{\infty}\left((-1)^{i+1} .2 i t^{0.5+2 i} E_{0.5,(1.5+2 i)}\left(-2 t^{0.5}\right)\right)=\sum_{i=1}^{\infty}\left((-1)^{i+1} .2 i t^{0.5+2 i} \sum_{k=0}^{\infty} \frac{\left(-2 t^{0.5}\right)^{k}}{(0.5 k+0.5+2 i) !}\right)
$$

The solution function $\varepsilon(t)$ and Fig. 3. below shows that, if viscoelasticity coefficient and stress function are known, then the value of stress will be obtained.

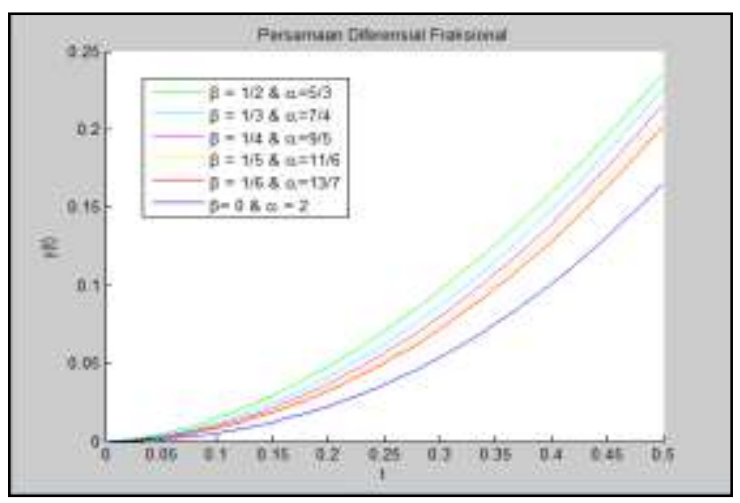

Fig. 2. Graph of Solution Function Case-2

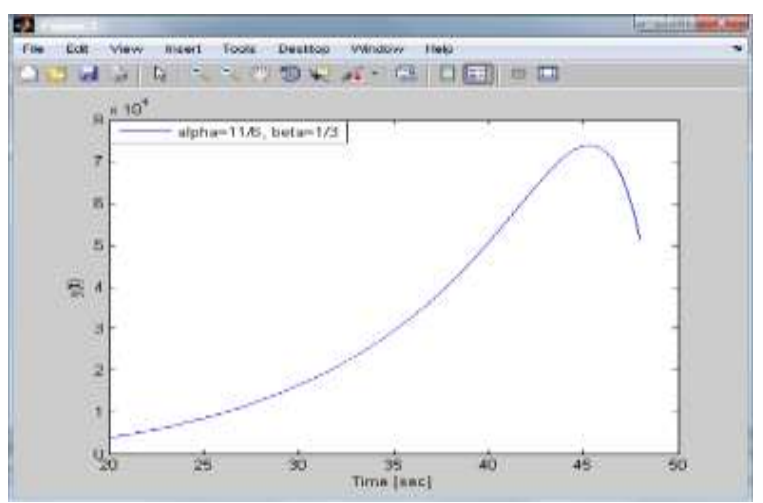

Fig. 3. Graph of solution: Strain Function 


\section{CONCLUSION}

Based on the research, the theory of fractional differential equations obtained the fact that if the numbers sequence of fractional-order $\left(\alpha_{n}\right)$ converges to $\alpha$, then the sequence of solution function $\left(y_{n}\right)$ also will converge to $y$. In the application, viscoelasticity problem is the relaxation problem that is a combination of fluid viscosity on Newtonian model problems and material elasticity on the Hooke's law in relation to stress and strain.

\section{ACKNOWLEDGMENT}

The authors thank to Rektor Universitas Padjadjaran and Director of Directorate of Research and Service Comunity Unpad who gave funding for dissemination of this paper. This work was fully supported by Universitas Padjadjaran under the Program of Penelitian Unggulan Perguruan Tinggi No. 718/UN6.3.1/PL/2017.

\section{REFERENCES}

[1] I. Petras, "Fractional derivatives, fractional integrals, and fractional differential equations in Matlab", Engineering education and research using MATLAB, InTech, 2011.

[2] D Matignon, "Generalized fractional differential and difference equations: stability properties and modelling issues", Mathematical Theory of Networks and Systems symposium, pp. 503-506, 1998.

[3] I. Podlubny, A. Chechkin, T Skovranek, Y. Q. Chen, and B. M. J Vinagre, "Matrix approach to discrete fractional calculus II : Partial fractional differential equations", Journal of Computational Physics, vol. 228, pp. 3137-3153, 2009.

[4] I. Podlubny, Fractional Differential Equations, Academic Press, San Diego, 1999.

[5] A. Loverro, Fractional calculus: history, definitions and applications for the engineer, Rapport technique, Univeristy of Notre Dame: Department of Aerospace and Mechanical Engineering, 2004, pp. 1-28.

[6] Y. Chen, I. Petras, D. Xue, "Fractional order control-a tutorial", American Control Conference, ACC'09, pp. 1397-1411, 2009.

[7] P. W. Ostalczyk, "A note on the Grünwald-Letnikov fractional-order backward-difference", Physica Scripta, vol. (T136), 2009.

[8] J. Wallner, "Existence of set-interpolating and energy-minimizing Curves", Computer Aided Geometric Design, vol. 21( 9), pp. 883-892, 2003.

[9] M. Hofer, H. Pottmann, "Designing energy-minimizing rigid body motions in the presence of obstacles", Tech. Rep. 142, Geometry Preprint Series, Vienna Univ. of Technology, 2005.

[10] H. Gunawan, F Pranolo, E. Rusyaman, "An interpolation method that minimizes an energy integral of fractional order", Proceeding of Asian Symposium of Computer Mathematics Singapore, pp. 151-161, 2007.

[11] Nicole Heymans and Igor Podlubny, "Physical interpretation of initial conditions for fractional differential equations with Riemann-Liouville fractional derivatives," Rheologica Acta Journal, vol. 45(5) , pp. 765$771,2006$.

[12] Alberto Carpinteri and Pietro Cornetti, "A fractional calculus approach to the description of stress and strain localization in fractal media", Chaos, Solitons \& Fractals Journal, vol. 13(1), pp. 85-94, 2002. 